\title{
Classical Novae as Supersoft X-ray Sources in the Andromeda Galaxy
}

\author{
Martin Henze $^{1}$, Wolfgang Pietsch ${ }^{1}$, Frank Haberl ${ }^{1}$, Margarita \\ Hernanz $^{2}$, Gloria Sala ${ }^{3}$, Massimo Della Valle ${ }^{4,5}$, Despina \\ Hatzidimitriou $^{6,7}$, Arne Rau ${ }^{1}$, Dieter H. Hartmann ${ }^{8}$, Vadim Burwitz ${ }^{1}$, \\ and Jochen Greiner ${ }^{1}$ \\ ${ }^{1}$ Max-Planck-Institut für extraterrestrische Physik, \\ Giessenbachstraße, D-85748 Garching, Germany \\ email: mhenze@mpe.mpg.de \\ ${ }^{2}$ Institut de Ciències de l'Espai (CSIC-IEEC), \\ Campus UAB, Fac. Ciències, E-08193 Bellaterra, Spain \\ ${ }^{3}$ Departament de Física i Enginyeria Nuclear, \\ EUETIB (UPC-IEEC), Comte d'Urgell 187, E-08036 Barcelona, Spain \\ ${ }^{4}$ INAF-Napoli, Osservatorio Astronomico di Capodimonte, \\ Salita Moiariello 16, I-80131 Napoli, Italy \\ ${ }^{5}$ International Centre for Relativistic Astrophysics, \\ Piazzale della Repubblica 2, I-65122 Pescara, Italy \\ ${ }^{6}$ Department of Astrophysics, Astronomy and Mechanics, Faculty of Physics, \\ University of Athens, Panepistimiopolis, GR15784 Zografos, Athens, Greece \\ ${ }^{7}$ Foundation for Research and Technology Hellas, IESL, Greece \\ ${ }^{8}$ Department of Physics and Astronomy, \\ Clemson University, Clemson, SC 29634-0978, USA
}

\begin{abstract}
We review the first results of a dedicated, on-going monitoring project for supersoft X-ray source (SSS) states of classical novae $(\mathrm{CNe})$ in the central region of the Andromeda galaxy (M 31). So far, during three high-cadence observation campaigns with the X-ray telescopes XMM-Newton and Chandra from 2006 until 2009 we detected $21 \mathrm{CNe}$, only four of which were seen in X-rays before. This increased the total number of known CNe with X-ray counterparts in M 31 to 60, which is significantly larger than for any other galaxy, including the Milky Way. We compiled a catalog of X-ray and optical properties for these 60 sources which allowed us to perform source statistics and population studies of CNe with SSS state in M 31 for the first time. Here we review the results of this analysis: (i) several correlations between X-ray and optical parameters, (ii) an analysis of the intrinsic fraction of CNe with SSS state, and (iii) the first nova population study in X-rays. Our first results are promising, however a continuing monitoring of the unique target M 31 is strongly needed to extend this valuable data base and to refine the early statements and predictions.
\end{abstract}

Keywords. galaxies: individual (M 31) — novae, cataclysmic variables - X-rays: binaries

\section{Introduction}

Classical novae (CNe) originate from thermonuclear explosions on white dwarfs (WDs) in cataclysmic binary systems (see e.g. Bode \& Evans 2008). Accreted hydrogen-rich material accumulates on the WD surface under degenerate conditions until hydrogen ignition starts a thermonuclear runaway. The resulting expansion of the hot envelope increases the optical brightness of the WD dramatically within days and leads to the ejection of mass at high velocities.

After the optical nova outburst, a fraction of the accreted material can remain on the WD surface under steady hydrogen burning (Starrfield et al. 1974). This powers a 
supersoft X-ray source (SSS) that becomes visible, once the opacity of the ejected matter reduces sufficiently (Starrfield 1989; Krautter 2002). These observational SSS turn-on time scales range from several weeks to even years. The duration of the SSS phase, typically years, is limited by the amount of hydrogen fuel left on the WD and the $S S S$ turn off marks the cease of the hydrogen burning.

Only X-ray observations are able to detect directly the hot WD photosphere and therefore provide important information on the physical parameters of the explosion, such as ejected and burned hydrogen mass (Sala \& Hernanz 2005; Hachisu \& Kato 2006). In the context of this symposium it should be emphasized that estimates of the accreted and ejected masses might help to determine the fraction of WDs in CNe which accumulate mass over time and ultimately can become Type Ia supernovae. Note, that for massive WDs, close to the Chandrasekhar limit, the expected SSS duration is very short $(<100$ d) (Tuchman \& Truran 1998; Sala \& Hernanz 2005).

The large spiral galaxy M 31 with its distance of only 780 kpc (Holland 1998; Stanek \& Garnavich 1998) and a total nova rate of $\sim 65 \mathrm{yr}^{-1}$ (Darnley et al. 2006) is a unique target for optical nova surveys (see Henze et al. 2008, and references therein). As of August 2011, more than 880 nova candidates have been found in M 31, more than twice as many as in the Galaxy. During the last decade, the observed nova rate was even larger by a factor of three for M 31 compared to the Galaxy (22 vs 7 novae $\mathrm{yr}^{-1}$ ), thanks to several monitoring projects (see e.g. Yuan et al. 2009; Pietsch 2010; Shafter et al. 2011; Kasliwal et al. 2011) and to the dedication of enthusiastic amateur observers. We maintain catalogs for optical novae in M 31 and other Local Group galaxies which are updated regularly (see http://www.mpe.mpg.de/ m31novae/opt/m31/index.php).

Whereas individual Galactic novae, because of their proximity, can be studied in great detail, the major advantage of M 31 is that many novae can be found in the field of view of one XMM-Newton or Chandra observation. The low Galactic foreground extinction $\left(N_{\mathrm{H}} \sim 6.7 \times 10^{20} \mathrm{~cm}^{-2}\right.$, Stark et al. 1992) favors a homogeneous X-ray monitoring of $\mathrm{CNe}$, all of which are effectively at the same distance. Therefore, M 31 is predestined for population studies of CNe and SSSs in a large spiral galaxy.

We initiated the first dedicated monitoring program for SSS states of CNe in the central region of M 31 using XMM-Newton EPIC and Chandra HRC-I. This study was motivated by new insights into the connections between M 31 SSSs and CNe based on analysis of archival data: Pietsch et al. (2005) found that optical novae constitute the major class of SSSs in the M 31 central region and Pietsch et al. (2007) discovered that the fraction of novae detected as SSSs was higher than previously estimated (>30\%). In this review, we describe the results of the first three monitoring campaigns.

\section{The $X M M$-Newton/Chandra Monitoring Program}

While the first campaign of our XMM-Newton/Chandra monitoring consisted of nine 20 ks pointings between June 2006 and March 2007 with a separation of 30 days (Henze et al. 2010), the second and third campaign used ten observations each from November to February 2007/8 and 2008/9, respectively, with an even higher cadence of ten days (Henze et al. 2011, hereafter HPH2011). The monitoring strategy was changed to account for a significant percentage of CNe in M 31 with short SSS phases found by Pietsch et al. (2007). In total, the exposure time for the three campaigns amounts to $\sim 160 \mathrm{~h}$. A stacked image of the XMM-Newton field of view of the M 31 central region is shown in Figure 1 and demonstrates the wealth of SSS counterparts of CNe in this region. 


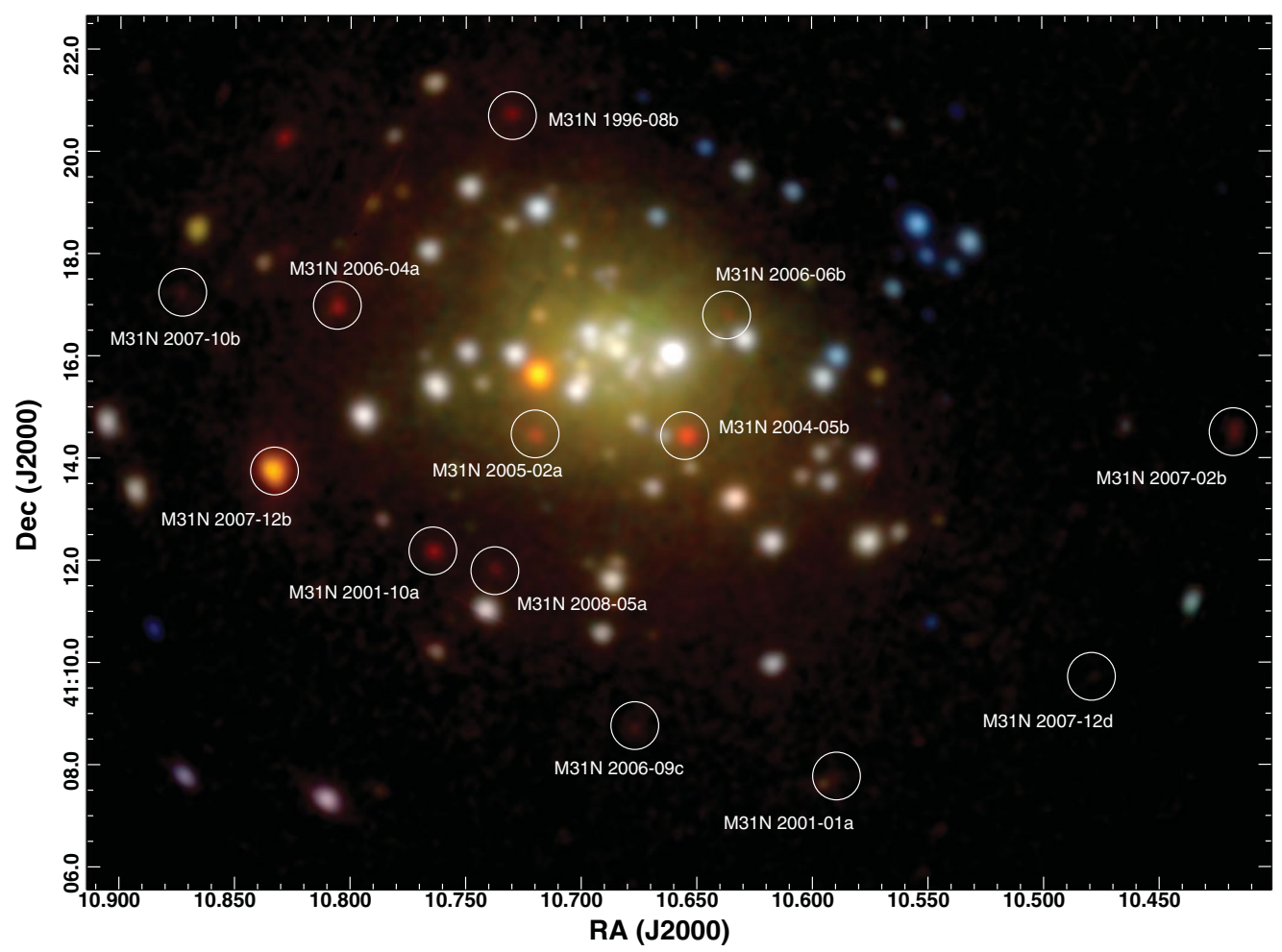

Figure 1. XMM-Newton EPIC RBG image of the central area of M 31 combining $300 \mathrm{ks}$ of pn, MOS1, and MOS2 each (2006-2009) in the (0.2-0.5), (0.5-1.0), and (1.0-2.0) keV bands. Red/orange colors indicate SSSs, CN counterparts are marked with white circles.

We discovered 17 new counterparts of CNe in M 31. An additional four known X-ray counterparts were still detected with long SSS durations. These results increased the number of CNe with an X-ray counterpart to 60 (about twice as many as known in the Galaxy) and added a significant number of X-ray measurements to the total sample. We compiled a catalog of optical and X-ray parameters for all 60 novae and used it to carry out the first source statistics and population studies of M 31 novae in X-rays. The results of this analysis are described in the following sections.

\section{Correlations between Nova Parameters}

We found four significant correlations between different optical and X-ray parameters which are shown in Figure 2 and described in the following. According to theoretical studies the CN properties depend mostly on the WD mass (see e.g. Livio 1992; Hachisu \& Kato 2006), which we assume is the main reason behind those relations.

SSS turn-on vs turn-off time. The two X-ray time scales of CNe are clearly correlated (Figure 2(a)). While it is not possible that $t_{\text {off }}<t_{\text {on }}$, we can place much stronger constraints on the correlation. The purple dashed line shows a corresponding relation derived by Hachisu \& Kato (2010), based on Galactic novae data, which is significantly different from our result. The reason for this is not yet understood. Additionally, Figure 2(a) shows a weakly significant ( $1 \sigma$ level) difference between novae belonging to the young (green) and old (blue) stellar population (see also Section 5). This result is strongly influenced by the small number of young novae. 

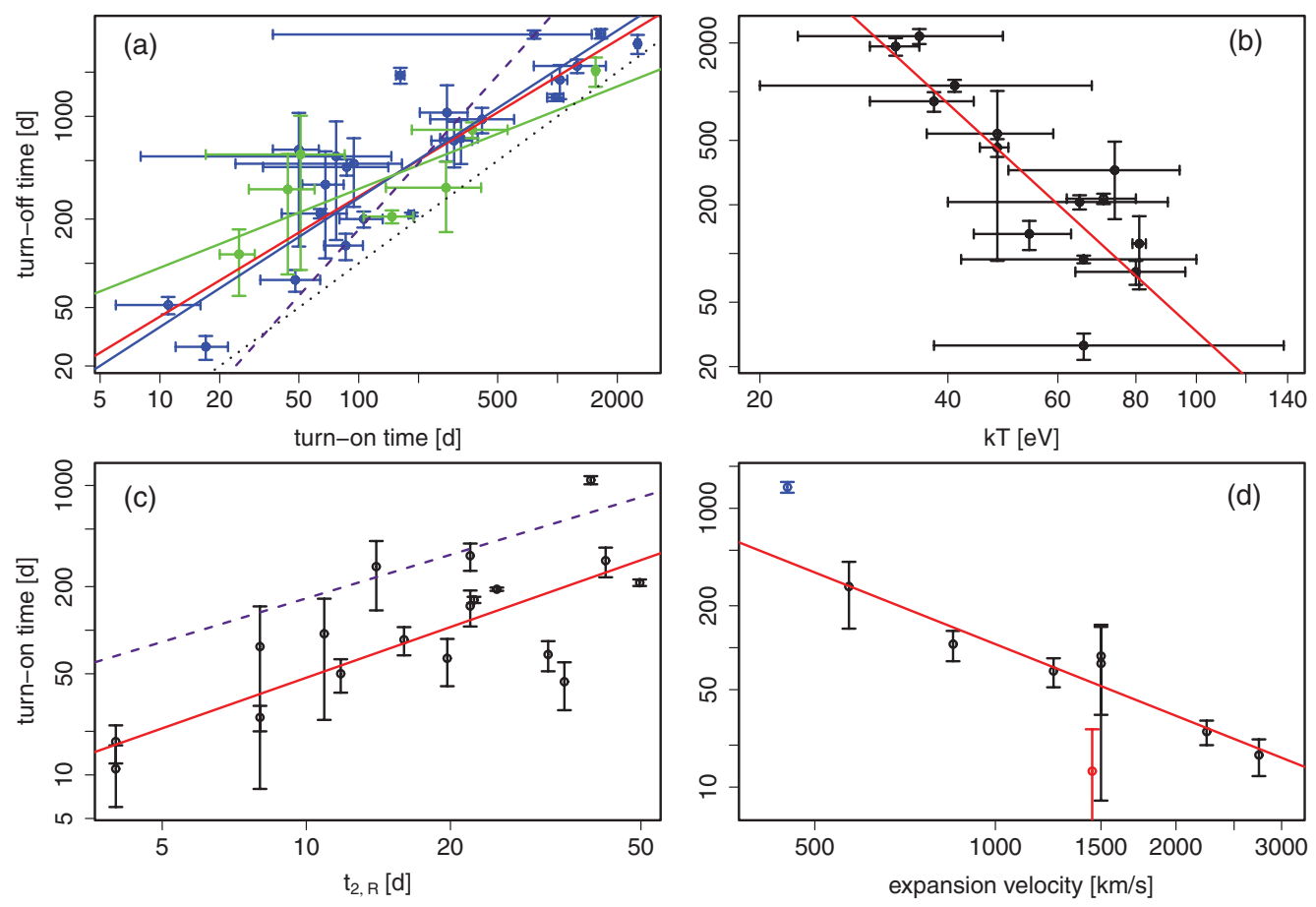

Figure 2. Correlations between nova parameters. Plots are double logarithmic. Red solid lines represent the best fits from weighted regressions, purple dashed lines show corresponding relations derived by Hachisu \& Kato (2010) for Galactic novae. Panels: (a) SSS turn-on vs turn-off times with $t_{\text {off }}=t_{\text {on }}$ marked by a black dotted line; green (blue) data and best fits refer to young (old) novae (see Section 5); (b) blackbody temperature vs SSS turn-off time; (c) optical $t_{2, R}$ decay time vs SSS turn-on time; (d) optical expansion velocity vs SSS turn-on time; red and blue data points were discarded for the fit. Credit: HPH2011, reproduced with permission (C)ESO.

SSS blackbody temperature vs SSS turn-off time. An anti-correlation exists between the blackbody temperature of the SSS, as measured from X-ray spectra, and $t_{\text {off: faster SSSs }}$ are hotter (see Figure 2(b)). Since the SSS temperature was linked to the WD mass in theoretical works (e.g. Sala \& Hernanz 2005), it appears likely that massive WDs produce fast and hot novae. Much has been written on the appropriateness of blackbody fits on SSS spectra of novae (see e.g. Kahabka \& van den Heuvel 1997; van Rossum \& Ness 2010; Ness et al. 2011). We fitted a sub-sample of X-ray spectra using the public NLTE WD atmosphere models by Rauch et al. (2010) and found that the results agree with blackbody fits for the scope of this analysis.

Optical decay time vs SSS turn-on time. Another correlation was found between the $t_{2, R}$ decay time of the optical light curve by 2 magnitudes in the $R$ band and the SSS $t_{\text {On }}$ time scale, suggesting that fast optical novae turn on fast as SSSs, too. However, the relatively large scatter around the best fit in Figure 2(c) points towards a more complex connection. Furthermore, there is a significant offset between our best fit and a similar relation found by Hachisu \& Kato (2010) for Galactic novae. This could be explained by their use of magnitudes from the emission-line free $y$ band, whereas in our case the $R$ band of course includes the $\mathrm{H} \alpha$ emission that is very prominent in nova spectra. Therefore, CN light curves are expected (and observed) to decline slower in the $R$ band, which is what we see in the plot. 
Expansion velocity vs SSS turn-on time. We found an anti-correlation between the expansion velocity of the ejected envelope from optical spectra and the SSS $t_{\text {On }}$ time (Figure $2(\mathrm{~d})$ ). This relation is intuitively clear: the faster the envelope expands the sooner it becomes transparent to (soft) X-rays. Factors like the metallicity and geometry of the ejecta also influence the $t_{\mathrm{On}}$ time, but the strong correlation suggests that they have less impact. We discarded two novae before fitting: M31N 2003-08c (blue), the X-ray luminosity of which is close to our sensitivity limit making its $t_{\mathrm{O}}$ uncertain, and M31N 2007-10a (red), which had an atypically low expansion velocity (Rau et al. 2007). This correlation connects both observational parameters needed to compute the ejected mass.

\section{Do All Novae Become SSSs?}

Based on theoretical models, a nova outburst is predicted to be followed by a phase of SSS visibility (Hachisu \& Kato 2010). However, only 20\% of optical novae in M 31 are observed in X-rays. This discrepancy could be an intrinsic effect, like self-absorption, or an observational bias caused by the inevitably incomplete monitoring coverage. The large sample of M 31 novae with SSS counterpart together with the large number of observations, allowed us to test the second scenario for the first time.

We conducted a simulation comparing predictions from theoretical models to our observed results. The starting point was a simple estimate of the observed WD mass distribution in CNe, based on Truran \& Livio (1986), which is strongly dominated by high-mass WDs due to their short recurrence time scales (see also Ritter et al. 1991). This was converted to a SSS turn-on time distribution using the models of Hachisu \& Kato (2006). Together with the SSS turn-off time, derived using the correlation described above, this resulted in a distribution of SSS durations.

Using a Monte Carlo Markov chain we randomly assigned SSS durations to $\sim 200$ optical novae found in the M 31 central region in 1995-2009. A CN was classified as "detected" if its SSS duration contained at least one of 48 X-ray observations (32 monitoring, 16 archival). Since nova SSSs are expected to radiate at the Eddington limit $\left(\sim 10^{37 . .38}\right.$ $\mathrm{erg} \mathrm{s}^{-1}$ ) during most of their lifetime a detection efficiency close to one is not unrealistic for our sensitivity limit of $\sim 10^{36} \mathrm{erg} \mathrm{s}^{-1}$. The chain then adjusted the intrinsic fraction of novae with SSS state so that the "detected" fraction matched the observed rate.

The result is shown in Figure 3: the distribution of the intrinsic fraction $x$ of novae with SSS state is close to 1 . We conclude that based on this simulation the X-ray nondetection of the majority of CNe in M 31 can be explained by an incomplete observational coverage.

\section{An X-ray Population Study}

The relatively large size of our catalog made it possible to conduct an analysis of different nova populations in X-rays. M 31 has played a key role in optical nova population studies (see e.g. Hatano et al. 1997; Shafter \& Irby 2001; Shafter et al. 2011), but in the X-ray regime no such analysis had been possible up to now. We applied two different methods, which are visualized in Figures 4 and 5 and will be described in the following.

Our first approach assumed that there are two different nova populations associated with the bulge and disk of M 31. Here the projected bulge of the galaxy (gray ellipse in Figure 4) is assumed to have an elliptic shape with a semi-major axis of $700^{\prime \prime}$, an ellipticity of 0.5 , and a position angle of $\sim 50^{\circ}$, according to NIR studies by Beaton et al. (2007). Novae within (outside of) this region are classified here as belonging to the old (young) stellar population of M 31 and marked by white (black) crosses in Figure 4. The 
only exception is the globular cluster nova M31N 2007-06b (Shafter \& Quimby 2007; Henze et al. 2009) which belongs to an old stellar population and is positioned outside the bulge ellipse. We compared the X-ray properties of both sub-samples and found that their blackbody temperatures are significantly different at the $88 \%$ confidence level, according to a t-test, with disk novae being on average hotter than bulge novae.

The second method defined two sub-samples of novae with high- and low-mass WDs, respectively, based on their SSS turn-on time and its connection to the WD mass as inferred from Hachisu \& Kato (2006). High-mass WDs have $M_{W D} \gtrsim 1.2 M_{\odot}$, which corresponds to $t_{o n} \lesssim 100 \mathrm{~d}$, and low mass WDs have $M_{W D} \lesssim 0.7 M_{\odot}$, corresponding to $t_{o n} \gtrsim 500 \mathrm{~d}$. This selection sampled both the high end of the WD mass distribution, which dominates the observed distribution in nova systems, and the region around the peak of the mass distribution of single WDs at $\sim 0.6 M_{\odot}$ (see e.g. Catalán et al. 2008, and references therein). Objects included in the high-mass (low-mass) WD subsamples are marked by blue (red) circles in Figure 4. We compared the de-projected distances from the M 31 center for both sub-sets in Figure 5(b), using the color coding as above. Again, both distributions are significantly different as confirmed by a KolmogorovSmirnoff test at the $95 \%$ confidence level. Novae with low-mass WDs appeared to show a stronger concentration to the center (bulge) of the galaxy that novae with high-mass WDs.

Of course, caveats included the high inclination of M 31 (77.5; e.g. Beaton et al. 2007), which suggests that projection effects might bias our results, as well as the small size of the sub-samples. However, we applied two different methods that utilized two different $\mathrm{X}$-ray parameters and both of them showed a first hint that there are differences in the X-ray regime between $\mathrm{CNe}$ in bulge and disk of $\mathrm{M} 31$. More observations are needed, in particular of the relatively neglected M 31 disk, to follow up these initial results.

\section{Summary \& Outlook}

In this review we described the first dedicated monitoring campaign for SSS counterparts of $\mathrm{CNe}$ in the central region of M 31. Three campaigns of XMM-Newton and Chandra observations have been analyzed so far and led to the discovery of $17 \mathrm{CNe}$ in X-rays. The high-cadence monitoring allowed us to detect rare objects and fast SSSs, thereby proving our monitoring strategy successful. We compiled and studied a homogeneous sample of $60 \mathrm{CNe}$ with X-ray properties. Using present-day observatories, such a project is only feasible for $\mathrm{M} 31$, owing to its proximity and large nova rate.

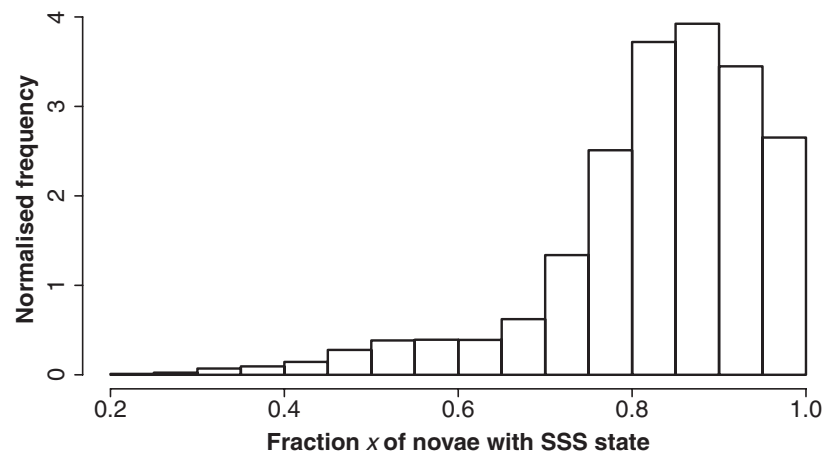

Figure 3. Simulated intrinsic fraction of M 31 novae with SSS state needed for the detection rate in our X-ray monitoring. Credit: HPH2011, reproduced with permission (C)ESO. 


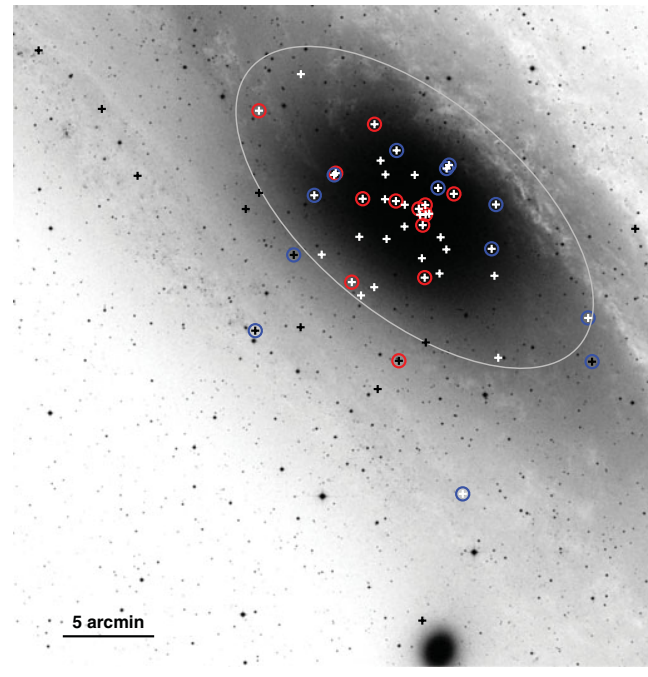

Figure 4. Positions of old (white) and young (black) novae with SSS counterpart in M 31 overlaid on a DSS2-R image. East is left and north is up. The gray ellipse indicates the boundary between bulge and disk region following Beaton et al. (2007). Red (blue) circles mark novae with low (high) mass white dwarfs, according to Section 5. Four of the 60 nova with X-ray emission are located outside this image.
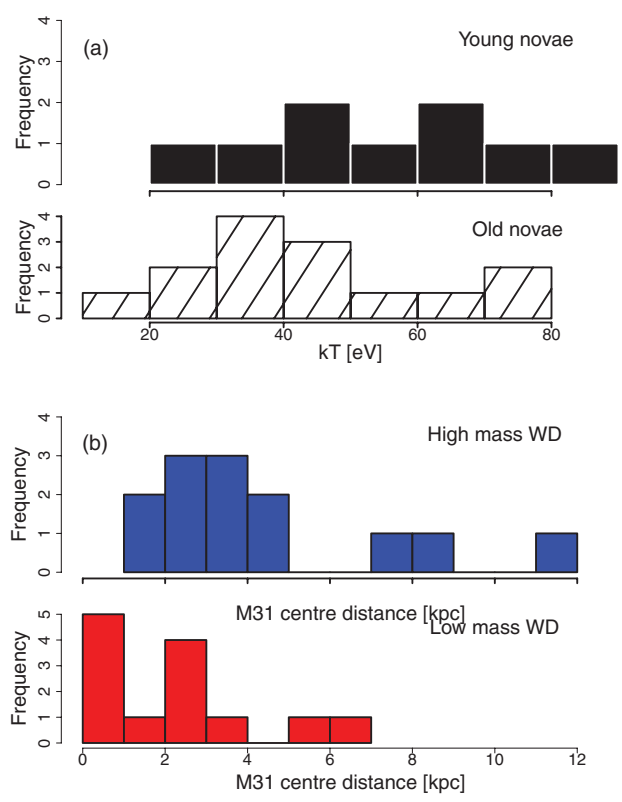

Figure 5. The distributions of (a) blackbody temperature for young and old novae and (b) de-projected M 31 center distance for novae with high- and low-mass WDs. Color coding is analogous to Figure 4. Credit: HPH2011, reproduced with permission (C)ESO.

Using the compiled nova catalog we conducted for the first time statistical studies of $\mathrm{CNe}$ properties in the X-ray range. These studies gave the following results: (a) several correlations connecting optical and X-ray parameters, (b) an analysis of the intrinsic SSS rate in $\mathrm{M} 31 \mathrm{CNe}$ based on theoretical models and the coverage of the monitoring, and (c) a population study of bulge vs disk novae based on their X-ray properties.

However, due to small number statistics many of the results described in this review only allowed a first glimpse into the physical connections that can be discovered using a dedicated monitoring of a nearby, high-nova rate galaxy. More observations are needed to confirm and further constrain our findings. Fortunately, as of August 2011 two more monitoring campaigns have already been executed in the winter of 2009/10 and 2010/11. The data analysis was partly finished and several new X-ray counterparts of CNe have been found. A further continuation of the monitoring program would guarantee a solid data base for statistical studies of classical novae in the X-ray regime.

\section{References}

Beaton, R. L., Majewski, S. R., Guhathakurta, P., et al. 2007, ApJ, 658, L91

Bode, M. F. \& Evans, A. 2008, Classical Novae, ed. Bode, M. F. \& Evans, A., Cambridge University Press

Catalán, S., Isern, J., García-Berro, E., \& Ribas, I. 2008, MNRAS, 387, 1693

Darnley, M. J., Bode, M. F., Kerins, E., et al. 2006, MNRAS, 369, 257

Hachisu, I. \& Kato, M. 2006, ApJS, 167, 59

Hachisu, I. \& Kato, M. 2010, ApJ, 709, 680

Hatano, K., Branch, D., Fisher, A., \& Starrfield, S. 1997, ApJ, 487, L45

Henze, M., Meusinger, H., \& Pietsch, W. 2008, A\&A, 477, 67 
Henze, M., Pietsch, W., Haberl, F., et al. 2010, A\&A, 523, A89

Henze, M., Pietsch, W., Haberl, F., et al. 2011, A\&A, 533, A52 [HPH2011]

Henze, M., Pietsch, W., Haberl, F., et al. 2009, A\&A, 500, 769

Holland, S. 1998, AJ, 115, 1916

Kahabka, P. \& van den Heuvel, E. P. J. 1997, ARA\&A, 35, 69

Kasliwal, M. M., Cenko, S. B., Kulkarni, S. R., et al. 2011, ApJ, 735, 94

Krautter, J. 2002, in American Institute of Physics Conference Series, Vol. 637, Classical Nova Explosions, ed. M. Hernanz \& J. José, 345

Livio, M. 1992, ApJ, 393, 516

Ness, J.-U., Osborne, J. P., Dobrotka, A., et al. 2011, ApJ, 733, 70

Pietsch, W. 2010, Astronomische Nachrichten, 331, 187

Pietsch, W., Fliri, J., Freyberg, M. J., et al. 2005, A\&A, 442, 879

Pietsch, W., Haberl, F., Sala, G., et al. 2007, A\&A, 465, 375

Rau, A., Burwitz, V., Cenko, S. B., et al. 2007, The Astronomer's Telegram, 1242, 1

Rauch, T., Orio, M., Gonzales-Riestra, R., et al. 2010, ApJ, 717, 363

Ritter, H., Politano, M., Livio, M., \& Webbink, R. F. 1991, ApJ, 376, 177

Sala, G. \& Hernanz, M. 2005, A\&A, 439, 1061

Shafter, A. W., Darnley, M. J., Hornoch, K., et al. 2011, ApJ, 734, 12

Shafter, A. W. \& Irby, B. K. 2001, ApJ, 563, 749

Shafter, A. W. \& Quimby, R. M. 2007, ApJ, 671, L121

Stanek, K. Z. \& Garnavich, P. M. 1998, ApJ, 503, L131

Stark, A. A., Gammie, C. F., Wilson, R. W., et al. 1992, ApJS, 79, 77

Starrfield, S. 1989, in Classical Novae, 39

Starrfield, S., Sparks, W. M., \& Truran, J. W. 1974, ApJS, 28, 247

Stiele, H., Pietsch, W., Haberl, F., et al. 2011 [arXiv:1106.4755]

Truran, J. W. \& Livio, M. 1986, ApJ, 308, 721

Tuchman, Y. \& Truran, J. W. 1998, ApJ, 503, 381

van Rossum, D. R. \& Ness, J. 2010, Astronomische Nachrichten, 331, 175

Yuan, F., Quimby, R. M., Chamarro, D. C., et al. 2009, Information Bulletin on Variable Stars, 5869,1

\section{Discussion}

Di Stefano: Concerning your statistical statements on bulge vs disk novae: Is your observational coverage of the M 31 bulge and disk homogeneous enough to prevent a bias?

Henze: The central field that we study has a homogeneous coverage and a significant part of it covers the disk. Some of the outer disk novae were discovered in the M 31 Large Survey (Stiele et al. 2011), but these observations were deep enough to avoid a bias towards SSS with high temperatures.

STARRFIELD: In your simulation of intrinsic SSS fraction, did you take into account that absorption within M 31 does attenuate SSSs much more than optical novae?

Henze: We studied the influence of M 31-intrinsic absorption, but due to the still relatively small number of novae with X-ray counterparts and to the spatial resolution of available extinction maps there is currently not enough information to describe qualitatively the impact on the SSS detection probability. 